\title{
Nickelates enter the scene
}

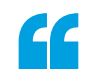

This motivated us to experimentally

characterize the similarities and differences between nickelate and cuprate superconductors
High-temperature superconductors hold promise for dissipationless transport of electrical current at relatively high temperatures. However, over 30 years after the first observation of high-temperature superconductivity in a copper-based oxide, or cuprate, there is still no consensus about the underlying mechanism. The recent observation of superconductivity in a nickel oxide, or nickelate, might help to understand such a mechanism. Chunjing Jia, Wei-Sheng Lee and colleagues, writing in Nature Materials, reveal that the electronic structure of the parent compound of nickelates - the material that, when doped, becomes superconducting is different from that of the parent compound of cuprates.

Nickelates were theoretically identified over 20 years ago as materials that could be engineered to display superconductivity, as they can reproduce some of the key properties of cuprates. Several theoretical proposals have motivated extensive experimental efforts to synthesize nickelate superconductors. Recently, these efforts culminated in the observation of superconductivity in the infinite-layer nickelate $\mathrm{NdNiO}_{2}$ doped with $\mathrm{Sr}$, which superconducts below $15 \mathrm{~K}$. This material has alternating neodymium and nickel oxide layers, thus it is isostructural to infinite-layer superconducting cuprates, which are made of copper oxide layers separated by spacer layers. The nickelate also nominally possesses the same number of $3 \mathrm{~d}$ electrons as the cuprates.

"However, whether the electronic structure of the newly discovered nickelate is similar to that of cuprates remained an open question," comments Matthias Hepting, first author of the new study. "This motivated us to experimentally characterize the similarities and differences between nickelate

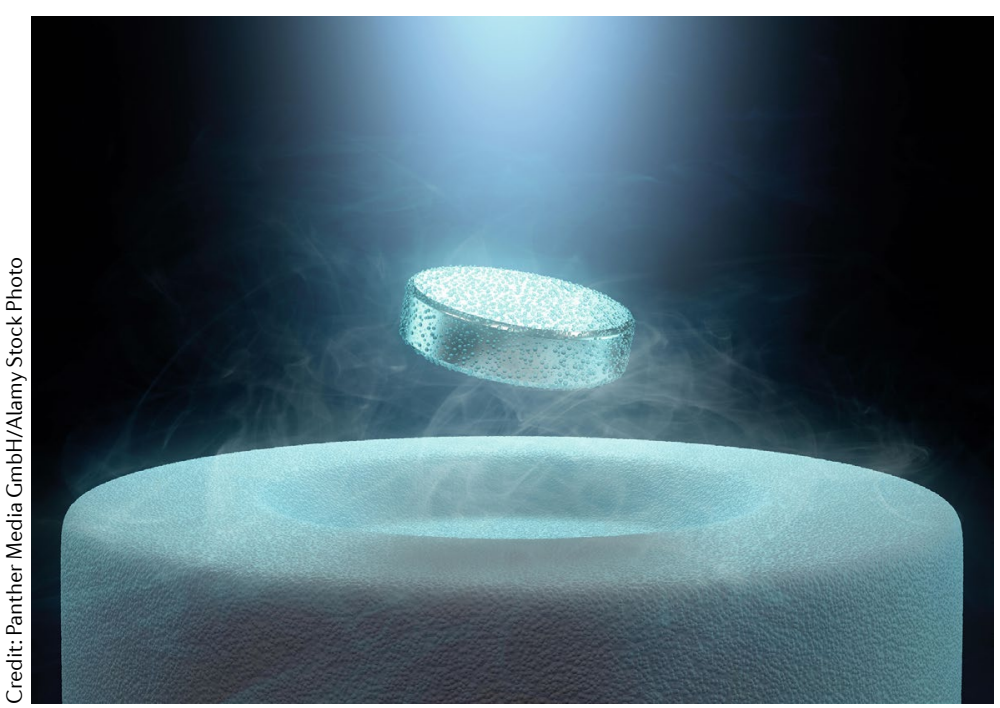

and cuprate superconductors." The authors used soft X-ray spectroscopy and density functional theory to study the electronic structure of $\mathrm{NdNiO}_{2}$ and a closely related material, $\mathrm{LaNiO}_{2}$. The results revealed that, despite the formal similarities between nickelates and cuprates, there are important differences in the electronic structures of these classes of materials.

Whereas in cuprates the transition-metal and oxygen electrons dominate the low-energy physics, in nickelates neodymium $5 \mathrm{~d}$ electrons also contribute to the low-energy electronic structure. As a consequence, the parent compound of nickelates is a $3 \mathrm{D}$ metal, in stark contrast with the $2 \mathrm{D}$ nature of the insulating parent compound of cuprates. "To our knowledge, the electronic structure we observed has not been seen in other transition metal oxides," says Jia. "It will provide a new playground for studying emergent phenomena in quantum materials."

The next step will be to investigate the evolution of the electronic structure when the system is doped with charge carriers. "We are also planning to study the difference between nickelates with different rare-earth elements," concludes Lee. "We hope that these pieces of information can further reveal the mechanism of superconductivity in the nickelates and help to discover other high-temperature superconductors."

Giulia Pacchioni

ORIGINAL ARTICLE Hepting, M. et al.

Electronic structure of the parent compound of superconducting infinite-layer nickelates. Nat. Mater. https://doi.org/10.1038/s41563-0190585-z (2020) RELATED ARTICLE Li, D. et al. Superconductivity in an infinite-layer nickelate. Nature 572, 624-627 (2019) 